\title{
Study of the Efficacy of Ksharaplota in Dushtavrana w.s.r to Infected Wound
}

\author{
Research Article
}

\section{Anantkumar V Shekokar ${ }^{*}$, Vijay P Ukhalkar²}

\author{
1. Ph.D Scholar, 2. Ph.D guide and Professor, Department of Shalyatantra, \\ Government Ayurved College, Nanded, Maharashtra, India
}

\begin{abstract}
Vrana ( wound and ulcer) \& process of healing is the soul of Shalyatantra Chikitsa (Surgical treatment ). Dushta Vrana means getting vitiated by Dosha \& hence Dushta Vrana or infected wound is characterized by bad smell, abnormal color with profuse discharge, severe pain and longer healing time The severity of infected wounds and their vast range of etiologies, The treatment is cleaning \& dressing the wound or it can be more extensive. It may require surgical intervention to close the wound and stabilize the patient. Acharya Sushruta defines Kshara as the substance possessing Ksharana and Kshanan properties. Ksharaplota formulation was selected and applied over the affected infected wound with the help of gauze piece. During the preparation of Ksharaplota, it is coated with Snuhi kshira, Apamarga kshara \& Haridra. All these drugs are Shodhana \& Ropana It was observed that Ksharaplota has the properties as sustained release of drug, absorbs discharges, less painful \& easily acceptable by the patient with excellent Sodhana \& Ropana Karma. The clinical study has been done on 438 patients, selected randomly and divided in two groups. Group A 220 patients i.e. trial group, were treated with local application of Ksharaplota. The Group B 218 patients i.e. control group, were treated with Gold standard. The clinical assessment was done on the basis of clinical presentation of Dushta Vrana, (Infected wound) before and after the treatment. As grading used for assessment of parameters which were ordinal in nature, "Wilcoxon Signed Ranks test" was used for within the group assessment (i.e. before and after treatment of a group). For between the group assessment of parameters, Mann Whitney - U test was applied. We had tested hypothesis for each parameter and result was interpreted accordingly. The level of significance was kept at $5 \%(\mathrm{P}=0.05)$. And the result of the present study found significant.
\end{abstract}

Key Words: Dushtavrana, Ksharaplota, Vranakovida, Infected Wound, Alkaline Medicated Gauze.

\section{Introduction}

Vrana and Shalyatantra are two terms that are analogous to each other and therefore considered two sides of the same coin. Vrana \& process of healing is the soul of Shalyatantra. Even in the definition of Shalyatantra, Sushruta emphasizes more on Vrana vinishchiyartha (1) which means Vrana Nidana, Lakashana, Samprapti, Uapadrava and Chikitsa. In the time of Sushruta, Vranakovida (2) was considered a type of super specialty for those who had expertise of diagnosis, prognosis \& management of Vrana which therefore stimulated research scholars to gain expertise in management of Vrana with different skilful modalities ( 3).

Dushta Vrana means getting vitiated by Dosha $\&$ hence Dushta Vrana or infected wound is characterized by bad smell, abnormal colour with

\section{* Corresponding Author:}

\section{Anantkumar V Shekokar}

Ph.D Scholar,

Department of Shalyatantra,

Government Ayurved College, Nanded,

Maharashtra. India

Email Id: dranantkumarshekokar@gmail.com profuse discharge, severe pain and longer healing time ( 4 ).Dushta Vrana or infected wounds are typically defined as cuts, lacerations, puncture, pathological in nature and cause damage to both skin \& underlying tissues (5)

General treatment process for infected wound is cleaning, using sterile saline solution \& extracting debris from the wound (6). An antibiotic gel is applied to prevent infection $\&$ then a sterile dressing is applied to keep the wound clean \& protected.(7) Usually patient avoids surgical debridement due to pain, fear \& psychological factors. Therefore there is scope for non surgical debridement modalities which are also known as parasurgical methods. Acharya Sushruta has mentioned Kshara (8) in Anu-shastra, Upayantra, Agropaharaniya and one of the Upakram of Vrana..

Local application of Ksharakarma i.e. Pratisarana. studies proved Shodhana \& Ropan properties of Kshara in the management of Dushta Vrana but during procedure of application (Avachuranan), there are some drawbacks while applying Kshara as it cannot be spread uniformly, very painful while spreading due to direct exposure, it can be used only for short duration, procedure of Kshara karma requires help of instruments and intervention by skill full medical person, absences of Vrana Strava Shoshana (absorption of discharge $\&$ necrotic tissues) $\&$ also the 
procedure is not easily tolerable and acceptable. Sushruta has given number of dressing materials (9) eg. Pichu, Plota, Kawalika \& Vikeshika. Among these Pichu is used not only to clean the Vrana but also to absorb the Vranastrava while Kawalika is used as dressing pad or just as to cover the wound. Plota (10) can be used to clean the Vrana as well as to cover the Vrana. It can also be used as Varti in those wounds or ulcers which are very deep to clean easily. In such wounds or ulcers Plotavarti removes all the debris hide inside the wounds or ulcers ( 11 ).

Snuhi kshira, Apamaraga kshara \& Haridra powder are used for the preparation of Ksharasutra (12) which is very popular throughout the world for the management of Fistula in ano for debridement of unhealthy granulation tissues. On the basis of excellent result shown by Ksharasutra, this formulation is selected and applied over the affected infected wound with the help of Alkaline medicateted gauze piece. During the prepartion of Ksharaplota, it is coated with Snuhi kshira, Apamarga kshara \& Haridra. All these drugs are Shodhana \& Ropana (13) in Karma that's why Ksharaplota is used for debridement or to remove unhealthy tissue and enhances formation of healthy granulation tissue to enhance quick normal epithelialization ( 14 ). In this regard it is important to note that a similar principal of healing has already been mentioned by Dalhana in Nimbhandhasangraha where Dalhana states that "After the purification(Shodhana) of wound there is no further need of treatment since the wound heals (Ropana) itself". (15)

It was observed that Ksharaplota has the properties as sustained release of drug, absorbs discharges, less painful \& easily acceptable by the patient with excellent Sodhana \& Ropana ( 16 ). Hence present study "Study The Efficacy Of Ksharaplota In Dushta Vrana w.s.r. to Infected Wound." was being conducted.

Thus in present study, an effort was made to establish the scientific validity of Ksharaplota in the management of Dushta Vrana and investigate its Shodhana (debridement) and Ropana (wound healing) properties.

\section{Materials and Methods}

Materials for trial group (A)

- Ksharaplota

- Bandage

\section{Preparation of Ksharaplota ( 17 )}

Ksharaplota was prepared at the Institute's department of Shalyatantra Following were the materials used for Ksharaplota preparation.

- Snuhikshira (Euphorbia nerifolia)

- Apamarg Kshara (Achyranthusaspera)

- Haridra churna ( Curcuma longa)

- Single layer Gauze piece

- Wooden Ring

- Cabinet

\section{Snuhi kshira (18)}

It was collected by taking an incision over the stem of Snuhi plant. The secreted milk (Kshir) was collected and stored in a pot. As Snuhi Kshira has a tendency to coagulate early, hence every time fresh Snuhi Kshira was taken.

\section{Apamarga Kshara (19)}

Whole Apamarga plant was to be taken and cut in pieces, after drying the plant in shade It was burned in light fire and ash was collected. The ash was dissolved in water, in the ratio 1:6. i.e. 1 part ash and 6 parts water. The solution, so formed was filtered with the help of percolator. Residual ash was again dissolved in 4 parts of water and the same procedure was repeated at least twice in order to take away all the alkaline material from the ash. Ultimately, the ash remains as a neutral residue, which should be thrown, the fluid was filtered several times (filtering once in a day) and finally, the Apamarga Kshara was obtained by evaporating the filtered solution.

\section{Haridra (20)}

Dry rhizomes of Haridra Plant were cut into pieces and powdered which was then sieved through a fine cloth. The fine powder thus obtained was stored in clean and sterile container.

\section{Gauze Piece}

According to Sushruta, gauze piece is similar to plota. It is a piece of woven surgical wool. A single layer gauze piece was used over a circular wooden ring having diameter of $23 \mathrm{~cm}$ to obtain the required Ksharaplota.

\section{Wooden ring}

A structure of double ring was used to hold the gauze piece.With the help of this ring, gauze piece was held tightly so that coating of Sunhi kshiria, Apamarga kshara \& Haridra was made very easy \& effective.

\section{Cabinet (21)}

It was used for drying the Ksharaplota. The prepared Ksharaplota on wooden rings was kept in a air tight cabinet for drying, sterilization \& storage purpose.

\section{Ksharaplota preparation}

A Round gauze piece, $23 \mathrm{~cm}$ in diameter was fixed over the doubled layered circular wooden ring. This gauze piece was made so tight that the coating can be done uniformly. Initially on $1^{\text {st }}$ day $50 \mathrm{ml}$ Sunhikshira was applied with the help of small cotton swab over the gauze on its entire circumference. The wet coated gauze with rings was placed inside the cabinet for drying for 1 day. On $2^{\text {nd }}$ day dried gauze was again smeared with $50 \mathrm{~m} 1$ Snuhikshira followed by 20 gms of Apamargakshara This was again dried in the cabinet for a day. On $3^{\text {rd }}$ day dried gauze was again smeared with $50 \mathrm{ml}$ Snuhikshira followed by $20 \mathrm{gms}$ Haridra churna. The prepared gauze was kept in the cabinet for drying (22). 
In this way the Ksharaplota was prepared having coating each of Snuhikshira $(150 \mathrm{ml})$ Apamargakshara (20 gms.) \& Haridra churna (20 gms.). The prepared Ksharaplota is then cut into $6 \times 6$ $\mathrm{cm}$. pieces and sealed in sterile polythene packs. Ksharaplota should be preferably kept in the cabinet for safety storage as well as sterilization.

\section{Materials for Control Group (B)}

- Normal Saline (N.S)

- Hydrogen Peroxide Solution $\left(\mathrm{H}_{2} \mathrm{O}_{2}\right)$

- Chlorinated Lime Water Boric Acid Solution (Eusol)

- Povidine - Iodine $0.5 \% \mathrm{w} / \mathrm{v}$ available Povidine (Betadine)

- Sterile Pad

- Bandage

\section{Inclusion Criteria}

- Patients having sign \& symptoms of DushtaVrana w.s.r.to traumatic lacerated infected wound over extremities.

- Within size upto length $6 \mathrm{~cm}$.and breadth $6 \mathrm{~cm}$.

- Patients of either sex will selected.

- The patients between the age group of 16-70 years.

\section{Exclusion Criteria}

- Anaemic patient $\mathrm{Hb}<10$ gm \%.

- Malnourish patient ( As per age, height \& weight proportion.)

- Bleeding disorders( Increased B.T \& C.T)

- Patient in septicemia

- The patients suffering from systemic disease such as AIDS, Tuberculosis, Diabetes mellitus, Hepatitis-B, Malignancy, Vericose ulcer, Deep vein thrombosis, Arterial ulcer, Neurogenic ulcer, Leprosy, Pregnancy, Acute \& Chronic renal failure, Jaundice \& cirrhosis of liver.

\section{Laboratory investigations}

- Hemoglobin percentage

- Total WBC count.

- Differential count.

- Erythrocyte sedimentation rate

- Platelet count

- B.T \& C.T

- Blood sugar level.

- Tridot test.

- Hepatitis -B (HbsAg)

- Sr. Creatinine.

- Urine sugar.

- Urine albumin.

- Urine microscopic examination.

\section{Criteria of Assessment \\ Pain}

Assessment will be done by Visual Analog

Scale.

\section{Daha(Burning Sensation)}

Assessment will be done by Visual Analog

Scale.

Kandu (Itching)

Scale.

Assessment will be done by Visual Analog

\section{Grade Description}

Amount of Srava (Discharge)

\begin{tabular}{|c|l|}
\hline 0 & No Strava / Dry Dressing \\
\hline 2 & $\begin{array}{l}\text { Gauze is slight moist } \\
\text { completely wet }\end{array}$ \\
\hline 3 & $\begin{array}{l}\text { Bandage is completely moist within } 24 \text { hours } \\
\text { but no need to change the dressing }\end{array}$ \\
\hline 4 & $\begin{array}{l}\text { Bandage is completely moist within } 24 \text { hours } \\
\text { and bandage is to be changed }\end{array}$ \\
\hline
\end{tabular}

\section{Consistency of Srava (Discharge)}

\begin{tabular}{|c|c|}
\hline 0 & Absent \\
\hline 1 & Prulent \\
\hline 2 & Mucopurulent \\
\hline 3 & Scanty \\
\hline 4 & Copious \\
\hline \multicolumn{2}{|c|}{ Gandha (Malodor) } \\
\hline 0 & Non Existence \\
\hline 1 & Minimum Bad Smell \\
\hline 2 & Mild Bad Smell \\
\hline 3 & Unpleasant Smell but tolerable \\
\hline 4 & Foul smell which is intolerable \\
\hline \multicolumn{2}{|c|}{ Wound Floor (Bed) } \\
\hline 0 & $\begin{array}{l}\text { Smooth Regular Floor and healthy } \\
\text { granulation tissue }\end{array}$ \\
\hline 1 & $\begin{array}{l}\text { Smooth regular floor with slight discharge } \\
\text { with absence of slough }\end{array}$ \\
\hline 2 & $\begin{array}{l}\text { Smooth irregular, slight discharge, less } \\
\text { granulation tissue and presence of slough }\end{array}$ \\
\hline 3 & $\begin{array}{l}\text { Rough floor and presence of slough with } \\
\text { moderate discharge }\end{array}$ \\
\hline 4 & $\begin{array}{l}\text { Rough irregular floor with more slough and } \\
\text { profuse discharge }\end{array}$ \\
\hline \multicolumn{2}{|c|}{ Tenderness } \\
\hline 0 & No tenderness \\
\hline 1 & Tenderness on palpation \\
\hline 2 & Tenderness on touch \\
\hline 3 & Unable to touch \\
\hline
\end{tabular}

\section{Wound Area}

- Surface area of wound measured by graph paper

- Healed Surface Area = x

- Actual Surface Area of wound on day $0=\mathrm{A}_{0}$

- Unhealed surface area remained on last day $=A_{L}$

- $\mathrm{X}=\mathrm{A}_{0}-\mathrm{A}_{\mathrm{L}}$

- Percentage of Healed Wound Area $=\left(X / A_{0}\right) \times 100$

\section{Unit Healing Time}

(Total number of days during Treatment) $/\left(\mathrm{A}_{0}-\mathrm{A}_{\mathrm{L}}\right)$ 


\section{Overall result}

\begin{tabular}{|l|c|} 
Class & $\begin{array}{l}\text { Pe r e n t g e o f } \\
\text { improvement (Total } \\
\text { relief in symptoms) }\end{array}$ \\
\hdashline Cured & $75-100 \%$ \\
Markedly improved & $50-74 \%$ \\
Moderately Improved & $25-49 \%$ \\
\hline Not Improved (Unchanged) & $00-24 \%$
\end{tabular}

\section{Trial group (A) - Ksharaplota}

- In this group the infected wound was washed with normal saline.

- Then the Ksharaplota was kept and bandaged with sterile dressing once in a day.

- On next day the Ksharaplota was changed.

- This procedure was done till the appearance of Shuddha Vrana Lakshanas or upto 14 days whichever was early.

\section{Control group (B) - Application of $\mathrm{H}_{2} \mathrm{O}$, Eusol, Povidine}

- In this group the infected wound was washed with Normal Saline.

- A local application of $\mathrm{H}_{2} \mathrm{O}_{2}$, Eusol, Povidine sequentially was then applied once in a day \& properly bandaged with sterile dressing.

- On the next day same procedure was repeated till the appearance of Shuddha Vrana Llakshanas or upto 14 days whichever was early.

- After 14 days or on attaining Shudhda Vrana Lakshanas (Sign of healing wound ) further management for both groups (Trial \& Control) will be only Normal Saline dressing followed by sterile bandaging which should done on alternate day till complete epithelialization was seen or for 1 month whichever was early.

\begin{tabular}{|c|c|c|c|}
\hline \multicolumn{4}{|c|}{ Table No. 1} \\
\hline $\begin{array}{l}\text { Sr. } \\
\text { No. }\end{array}$ & Particulars & $\begin{array}{c}\text { Trial Group } \\
\text { (A) }\end{array}$ & $\begin{array}{c}\text { Control } \\
\text { Group (B) }\end{array}$ \\
\hline 1 & Drug & Ksharaplota & $\begin{array}{l}\mathrm{H}_{2} \mathrm{O} \text {, Eusol, } \\
\text { Povidine }\end{array}$ \\
\hline 2 & Route & Local & Local \\
\hline 3 & Frequency & Once Daily & Once Daily \\
\hline 4 & Duration & $\begin{array}{l}\text { 14 Days or till } \\
\text { appearance of } \\
\text { Shuddha } \\
\text { Vrana } \\
\text { Lakshanas }\end{array}$ & $\begin{array}{l}\text { 14 Days or till } \\
\text { appearance of } \\
\text { Shuddha } \\
\text { Vrana } \\
\text { Lakshanas }\end{array}$ \\
\hline 5 & $\begin{array}{l}\text { Sample } \\
\text { size }\end{array}$ & 220 & 218 \\
\hline
\end{tabular}

\section{Observation of the Study Gender}

Out of 218 patients in control group, 118 $(54.13 \%)$ were male and $100(45.87 \%)$ patients were female. In trial group, out of 220 patients $116(52.73 \%)$ patients were male while $104(47.27 \%)$ were female.
The Age group of the selected cases for the study was 16-70 years divided into 4 age groups viz. 16-30 years, 30-45 years, 45-60 years and Above 60 years. Majority of the 241 cases i.e. $55 \%$ were between the age group of 30-45 years. In control group, out of 218 patients, $116(53.21 \%)$ were from 30 to 45 years, $60(27.52 \%)$ patients were from age group 16 to 30 years, $40(18.35 \%)$ patients from 45 to 60 years while $02(00.92 \%)$ belongs to age group 60 years \& above. In trial group, out of 220 patients, $125(56.82 \%)$ were from 30 to 45 years, $53(24.09 \%)$ patients were from age group 16 to 30 years, $40(18.18 \%)$ patients from 45 to 60 years while $02(00.91 \%)$ belongs to age group 60 years $\&$ above.

\section{Marital status}

Regarding marital status, from control group, out of 218 patients, 159 (72.94\%) were married while $59(27.06 \%)$ were unmarried. Similarly from trial group, out of 220 patients $161(73.18 \%$ ) were married while $59(26.82 \%)$ were unmarried.

\section{Religion}

Regarding religion wise distribution of cases from control group, out of 218 patients, $187(85.78 \%)$ were Hindu, 14(06.42\%) were Muslim, $11(05.05 \%)$ patients from Christian religion while $06(02.75 \%)$ belongs to Buddha. Similarly from trial group, out of 220 patients, $189(85.91 \%)$ were Hindu, 13( 5.91\%) were Muslim, $11(05.00 \%)$ patients from Christian religion while $07(03.18 \%)$ belongs to Buddhist community. Thus maximum patients belonged to Hindu religion which might be due to the dominance of Hindu religion in the society.

\section{Socio-economic status}

In control group, out of 218 patients, 139 $(63.76 \%)$ patients were from middle class, $35(16.06 \%)$ were from rich class, while $44(20.18 \%)$ belonged to poor class. Similarly in trial group, out of 220 patients, $143(65 \%)$ patients were from middle class, $34(15.45 \%)$ were from rich class, while $43(19.55 \%)$ belongs to poor class Thus, maximum patients belonged to middle class.

\section{Educational status}

In control group, out of 218 patients, 45 (20.64\%) patients were illiterate, 63(28.90\%) had primary education, $93(42.66 \%)$ patients completed their graduation while $17(07.80 \%)$ had done their post graduation. From trial group, out of 220 patients, 44 $(20.00 \%)$ patients were illiterate, $62(28.18 \%)$ had primary education, $97(44.09 \%)$ patients completed their graduation while $17(07.73 \%)$ completed their post graduation.

\section{Occupation}

In control group, out of 218 patients, 41 $(18.80 \%)$ patients were students, $28(12.84 \%)$ were labour, $27(12.39 \%)$ patients were government employee, $38(17.43 \%)$ were from agricultural field, 46 $(21.10 \%)$ were house wives, $19(08.72 \%)$ patients were 
farmers while $19(08.72 \%)$ belonged to other occupations. From trial group, out of 220 patients, 44 (20\%) patients were students, 27 (12.27\%) were labour, $28(12.73 \%)$ patients were government employee, 38 $(17.27 \%)$ were from agricultural field, 41 (18.64\%) were house wives, $24(19.91 \%)$ patients were farmer while $18(08.18 \%)$ belonged to other occupations.

\section{Sleep}

In control group, out of 218 patients, 138 (63.30\%) patients had good sleep, 51(23.39\%) were had sound sleep, while $29(13.30 \%)$ were had disturbed sleep. In trial group, out of 220 patients, 139(68.18\%) patients had good sleep, 53(24.09\%) had sound sleep, while $28(12.73 \%)$ were having disturbed sleep.

\section{Deha prakruti}

In control group, out of 218 patients, 83 (38.07\%) patients were of Vata-pittaj prakruti, 91(41.74\%) were of Kapha-pittaj prakruti, while 44(20.18\%) were having Vata-kaphaj prakruti. In trial group, out of 220 patients, 88 (40\%) patients were of Vata-pittaj prakruti, $88(40 \%)$ were having Kapha-pittaj prakruti, while 44(20\%) had Vata-kaphaj prakruti.

\section{Manas Prakruti}

In control group, out of 218 patients, 104 (47.71\%) patients were having Rajasik prakruti,
114(52.29\%) were having Tamasik prakruti, while no patient belonged to Satvik prakruti In trial group, out of 220 patients, $106(48.18 \%)$ patients were having Rajasik prakruti, 114(51.82\%) were having Tamasik prakruti, while no patient belonged to Satvik prakruti.

\section{Sarata}

In control group, out of 218 patients, 25(14.47\%) patients were having Pravara sarata, $121(55.50 \%)$ had Madhya sarata, while72 (33.03\%) patients had Avar sarata.

In trial group, out of 220 patients, 26(11.82\%) patients had Pravara sarata, 123 (55.91\%) had Madhya sarata, while71 (32.27\%) patients had Avar sarata.

\section{Result of the Study}

As grading used for assessment of parameters which were ordinal in nature, "Wilcoxon Signed Ranks test" was used for within the group assessment (i.e. before and after treatment of a group). For between the group assessment of parameters, Mann Whitney - U test was applied.

We had tested hypothesis for each parameter and result was interpreted accordingly. The level of significance was kept at $5 \%(\mathrm{P}=0.05)$. Proper summary statistics like mean, mean difference, median difference were provided along with graphs and diagrams.

Table 2: Effect on Vedana

\begin{tabular}{|c|c|c|c|c|c|c|c|c|}
\hline Vedana & \multicolumn{2}{|c|}{ Mean Score } & $\begin{array}{c}\text { Median } \\
\text { Difference }\end{array}$ & $\begin{array}{c}\text { Sample } \\
\text { size }\end{array}$ & $\begin{array}{c}\text { Wilcoxon signed } \\
\text { rank test (T Value) }\end{array}$ & P value & Inference \\
\hline $\begin{array}{c}\text { Control } \\
\text { Group }\end{array}$ & 7.546 & 3.959 & 3.587 & 4 & 218 & $(\mathrm{~T}+)=23871$ & $\mathrm{P}<0.0001$ & $\begin{array}{c}\text { Extremely } \\
\text { significant }\end{array}$ \\
\hline $\begin{array}{c}\text { Trial } \\
\text { Group }\end{array}$ & 7.568 & 3.955 & 3.614 & 4 & 220 & $\begin{array}{c}(\mathrm{T}+)=24310 \\
(\mathrm{~T}-)=0\end{array}$ & $\mathrm{P}<0.0001$ & $\begin{array}{c}\text { Extremely } \\
\text { significant }\end{array}$ \\
\hline
\end{tabular}

Table 3: Effect on Daha

\begin{tabular}{|c|c|c|c|c|c|c|c|c|}
\hline \multirow{2}{*}{ Daha } & \multicolumn{2}{|c|}{ Mean Score } & $\begin{array}{c}\text { Median } \\
\text { Difference }\end{array}$ & $\begin{array}{c}\text { Sample } \\
\text { size }\end{array}$ & $\begin{array}{c}\text { Wilcoxon signed } \\
\text { rank test }\end{array}$ & P value & Inference \\
\hline $\begin{array}{c}\text { Control } \\
\text { Group }\end{array}$ & 6.977 & 3.867 & 3.110 & 3 & 218 & $\begin{array}{c}(\mathrm{T}+)=23871 \\
(\mathrm{~T}-)=0\end{array}$ & $\mathrm{P}<0.0001$ & $\begin{array}{c}\text { Extremely } \\
\text { significant }\end{array}$ \\
$\begin{array}{c}\text { Trial } \\
\text { Group }\end{array}$ & 6.982 & 3.877 & 3.105 & 3 & 220 & $\begin{array}{c}(\mathrm{T}+)=24310 \\
(\mathrm{~T}-)=0\end{array}$ & $\mathrm{P}<0.0001$ & $\begin{array}{c}\text { Extremely } \\
\text { significant }\end{array}$ \\
\hline
\end{tabular}

Table 4: Effect on Tenderness

\begin{tabular}{|c|c|c|c|c|c|c|c|c|}
\hline \multirow{2}{*}{ Tenderness } & \multicolumn{3}{|c|}{ Mean Score } & $\begin{array}{c}\text { Median } \\
\text { Difference }\end{array}$ & $\begin{array}{c}\text { Sample } \\
\text { size }\end{array}$ & $\begin{array}{c}\text { Wilcoxon signed } \\
\text { rank test (T Value) }\end{array}$ & P value & Inference \\
\hline Control Group & 2.564 & 0.9725 & 1.592 & 2 & 218 & $\begin{array}{c}(\mathrm{T}+)=23871 \\
(\mathrm{~T}-)=0\end{array}$ & $\mathrm{P}<0.0001$ & $\begin{array}{l}\text { Extremely } \\
\text { significant }\end{array}$ \\
\hline Trial Group & 2.564 & 1.014 & 1.550 & 2 & 220 & $\begin{array}{c}(\mathrm{T}+)=24090 \\
(\mathrm{~T}-)=0\end{array}$ & $\mathrm{P}<0.0001$ & $\begin{array}{l}\text { Extremely } \\
\text { significant }\end{array}$ \\
\hline
\end{tabular}




\section{Table 5: Effect on Kandu}

\begin{tabular}{|c|c|c|c|c|c|c|c|c|} 
Kandu & BT & AT & Diff. & $\begin{array}{c}\text { Median } \\
\text { Difference }\end{array}$ & $\begin{array}{c}\text { Sample } \\
\text { size }\end{array}$ & $\begin{array}{c}\text { Wilcoxon signed } \\
\text { rank test (T } \\
\text { Value) }\end{array}$ & P value & Inference \\
$\begin{array}{c}\text { Control } \\
\text { Group }\end{array}$ & 1.99 & 0.9725 & 1.018 & 1 & 218 & $\begin{array}{c}(\mathrm{T}+)=23871 \\
(\mathrm{~T}-)=0\end{array}$ & $\mathrm{P}<0.0001$ & $\begin{array}{c}\text { Extremely } \\
\text { significant }\end{array}$ \\
$\begin{array}{c}\text { Trial } \\
\text { Group }\end{array}$ & 1.986 & 0.9682 & 1.018 & 1 & 220 & $\begin{array}{c}(\mathrm{T}+)=23871 \\
(\mathrm{~T}-)=0\end{array}$ & $\mathrm{P}<0.0001$ & $\begin{array}{c}\text { Extremely } \\
\text { significant }\end{array}$ \\
\hline
\end{tabular}

Table 6: Effect on Discharge

\begin{tabular}{|c|c|c|c|c|c|c|c|c|} 
Discharge & \multicolumn{2}{|c|}{ Mean Score } & $\begin{array}{c}\text { Median } \\
\text { Difference }\end{array}$ & $\begin{array}{c}\text { Sample } \\
\text { size }\end{array}$ & $\begin{array}{c}\text { Wilcoxon signed } \\
\text { rank test (T Value) }\end{array}$ & P value & Inference \\
$\begin{array}{c}\text { Control } \\
\text { Group }\end{array}$ & 2.408 & 0.6697 & 1.739 & 2 & 218 & $\begin{array}{c}(\mathrm{T}+)=23871 \\
(\mathrm{~T}-)=0\end{array}$ & $\mathrm{P}<0.0001$ & $\begin{array}{c}\text { Extremely } \\
\text { significant }\end{array}$ \\
\hline Trial Group & 2.41 & 0.6727 & 1.736 & 2 & 220 & $\begin{array}{c}(\mathrm{T}+)=24310 \\
(\mathrm{~T}-)=0\end{array}$ & $\mathrm{P}<0.0001$ & $\begin{array}{c}\text { Extremely } \\
\text { significant }\end{array}$ \\
\hline
\end{tabular}

Table 7. Effect on Gandha

\begin{tabular}{|c|c|c|c|c|c|c|c|c|}
\hline Gandha & \multicolumn{3}{|c|}{ Mean Score } & Median & Sample & $\begin{array}{c}\text { Wilcoxon } \\
\text { signed rank } \\
\text { test (T Value) }\end{array}$ & P value & Inference \\
\hline $\begin{array}{c}\text { Control } \\
\text { Group }\end{array}$ & 2.092 & 0.01376 & 2.078 & 2 & 218 & $\begin{array}{c}(\mathrm{T}+)=23871 \\
(\mathrm{~T}-)=0\end{array}$ & $\mathrm{P}<0.0001$ & $\begin{array}{c}\text { Extremely } \\
\text { significant }\end{array}$ \\
\hline Trial Group & 2.091 & 0.01364 & 2.077 & 2 & 220 & $\begin{array}{c}(\mathrm{T}+)=24310 \\
(\mathrm{~T}-)=0\end{array}$ & $\mathrm{P}<0.0001$ & $\begin{array}{c}\text { Extremely } \\
\text { significant }\end{array}$ \\
\hline
\end{tabular}

Table 8: Effect on Wound floor bed

\begin{tabular}{|c|c|c|c|c|c|c|c|c|}
\hline Wound floor & BT & AT & Diff. & $\begin{array}{c}\text { Median } \\
\text { Difference }\end{array}$ & $\begin{array}{c}\text { Sample } \\
\text { size }\end{array}$ & $\begin{array}{c}\text { Wilcoxon } \\
\text { signed rank } \\
\text { test (T Value) }\end{array}$ & P value & Inference \\
\hline $\begin{array}{c}\text { Control } \\
\text { Group }\end{array}$ & 2.986 & 0.0092 & 2.98 & 3 & 218 & $\begin{array}{c}(\mathrm{T}+)=23871 \\
(\mathrm{~T}-)=0\end{array}$ & $\mathrm{P}<0.0001$ & $\begin{array}{c}\text { Extremely } \\
\text { significant }\end{array}$ \\
\hline $\begin{array}{c}\text { Trial Group } \\
\text { Srial }\end{array}$ & 2.991 & 0.0091 & 2.982 & 3 & 220 & $\begin{array}{c}(\mathrm{T}+)=24310 \\
(\mathrm{~T}-)=0\end{array}$ & $\mathrm{P}<0.0001$ & $\begin{array}{c}\text { Extremely } \\
\text { significant }\end{array}$ \\
\hline
\end{tabular}

Table 9: Effect on Consistency of discharge

\begin{tabular}{|c|c|c|c|c|c|c|c|c|}
\hline \multirow{2}{*}{$\begin{array}{l}\text { Consistency of } \\
\text { Discharge }\end{array}$} & \multicolumn{3}{|c|}{ Mean Score } & \multirow{2}{*}{$\begin{array}{c}\text { Median } \\
\text { difference }\end{array}$} & \multirow{2}{*}{$\begin{array}{c}\text { Sample } \\
\text { size }\end{array}$} & \multirow{2}{*}{$\begin{array}{c}\text { Wilcoxon } \\
\text { signed rank } \\
\text { test(T Value) }\end{array}$} & \multirow[b]{2}{*}{$P$ value } & \multirow[b]{2}{*}{ Inference } \\
\hline & BT & AT & Diff. & & & & & \\
\hline Control Group & 2.99 & 0.0092 & 2.965 & 3 & 218 & $\begin{array}{c}(\mathrm{T}+)=10296 \\
(\mathrm{~T}-)=0\end{array}$ & $\mathrm{P}<0.0001$ & $\begin{array}{l}\text { Extremely } \\
\text { significant }\end{array}$ \\
\hline Trial Group & 2.991 & 0.0091 & 2.98 & 3 & 220 & $\begin{array}{c}(\mathrm{T}+)=24310 \\
(\mathrm{~T}-)=0\end{array}$ & $\mathrm{P}<0.0001$ & $\begin{array}{l}\text { Extremely } \\
\text { significant }\end{array}$ \\
\hline
\end{tabular}

Table 10: Effect on Wound Area

\begin{tabular}{|l|l|l|l|l|l|l|l|l|} 
Wound Area & \multicolumn{2}{|l|}{ Mean Score } & $\begin{array}{l}\text { Median } \\
\text { Difference }\end{array}$ & $\begin{array}{l}\text { Sample } \\
\text { size }\end{array}$ & $\begin{array}{l}\text { Wilcoxon signed } \\
\text { rank test (T Value) }\end{array}$ & P value & Inference \\
\hline $\begin{array}{l}\text { C o n t r o l } \\
\text { Group }\end{array}$ & 2.266 & 0.9862 & 1.28 & 1 & 218 & $\begin{array}{l}\text { (T+)=23871 } \\
(\mathrm{T}-)=0\end{array}$ & $\mathrm{P}<0.0001$ & $\begin{array}{l}\text { Extremely } \\
\text { significant }\end{array}$ \\
\hline Trial Group & 2.268 & 0.9909 & 1.277 & 1 & 220 & $\begin{array}{l}(\mathrm{T}+)=24310 \\
(\mathrm{~T}-)=0\end{array}$ & $\mathrm{P}<0.0001$ & $\begin{array}{l}\text { Extremely } \\
\text { significant }\end{array}$ \\
\hline
\end{tabular}




\begin{tabular}{|c|c|c|c|c|c|c|c|c|}
\hline \multicolumn{9}{|c|}{ Table 11. Unit Healing Time } \\
\hline Unit Hea & Mean & core & & Median & Sample & 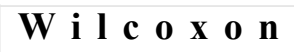 & & \\
\hline Time & BT & AT & Diff. & $\begin{array}{l}\text { Differe } \\
\text { nce }\end{array}$ & size & $\begin{array}{l}\text { signed rank test } \\
\text { (T Value) }\end{array}$ & P value & Inference \\
\hline Control Group & 1.995 & & 1.009 & 然 & 218 & $\begin{array}{l}(\mathrm{T}+)=23653 \\
(\mathrm{~T}-)=0\end{array}$ & $\mathrm{P}<0.0001$ & $\begin{array}{l}\text { Extremely } \\
\text { significant }\end{array}$ \\
\hline Trial Group & 1.99 & 1.005 & 0.99 & 1 & 220 & $\begin{array}{l}(\mathrm{T}+)=23871 \\
(\mathrm{~T}-)=0\end{array}$ & $\mathrm{P}<0.0001$ & $\begin{array}{l}\text { Extremely } \\
\text { significant }\end{array}$ \\
\hline
\end{tabular}

\section{Overall Result}

Table No. 12: Showing the overall result

\begin{tabular}{|c|c|c|c|c|}
\hline \multirow{2}{*}{ Overall Effect } & Control Group & Trial Group & Number Of Patients & $\%$ \\
\cline { 2 - 5 } & Number Of Patients & $\mathbf{\%}$ & 0 & 0 \\
\hline Cured & 1 & 0.46 & 220 & 100 \\
\hline Markedly improved & 217 & 99.54 & 0 & 0 \\
\hline Moderately improved & 0 & 0 & 0 & 0 \\
\hline TOTProved (Unchanged) & 0 & 0 & $\mathbf{2 2 0}$ & $\mathbf{1 0 0}$ \\
\hline
\end{tabular}

From control group out of 218 patients in the study 1 patient i.e. $(0.46 \%)$ was totally cured while 217 i.e. $(99.54 \%)$ patients were had marked improvement in their complaints. Similarly from trial group out of 220 i.e. $(100 \%)$ patients, had marked improvement in their complaints.

\section{Discussion}

Ksharaplota composed of Snuhi \& Apamaraga has Ksharan and Kshanan properties, Apamargakshara also cauterize tissue by its Ksharana Guna while Haridra offers Krimighana \& Ropana properties thus provides VranaShodhana \& VranaRopana effect. Chemical properties of Ksharaplota remove unhealthy tissue and alkaline nature of Kshar offers aseptic and antimicrobial effect thus reduces chance of secondary infection. Snuhi component of Ksharaplota provides moisturizing effect due to its sticky property which ultimately promotes epidermal migration resulting enhanced synthesis of connective tissue. The Snuhi latex having binding property therefore retains surgical benefits (debridement, scrapping, hemostatic, aseptic, antiseptic and healing effect) of Apamargakshara for longer time. Snuhikshira, Apamargakshara and Haridra support biological action of each other and not contraindicated when applied together. Sustained release action of Ksharaplota medication releases ingredient slowly and make available medicine for longer duration to achieve complete healing of chronic wound.

Ksharaplota overall possess penetrating, scrapping, draining, debridement, sclerosing, healing, antibacterial and anti inflammatory effect in the management of wound and cuts. All three ingredients contribute significantly towards mode of action as follows ( 23 ).

Snuhi (Latex of Euphorbia nerifolia) Kshira possess properties such as; Lekhana, Vedanasthapana, Rakthashodhak and Vishaghana due to Katu \& Tikshana Rasa and Ushna Virya. The latex of plant also found to have rubifacient, irritant and powerful caustic action when applied to a raw surface; it also offers analgesic, anti-inflammatory, wound healing and antibacterial activity. It is believe that Euphorbia neriifolia enhances wound healing process by promoting tensile strength, epithelization and angiogenesis.

Apamarga (Achyranthus aspera) Kshara offers Lekhana, Chedana, Bhedana, Ropana, Kushtaghna, Shodhana and Krumighana properties due to Katu \& lavana Rasa and Ushna Virya. Katu Rasa contributes towards Shonit

Sanghat Bhinnati action of Apamarg Kshara which breakdown pus pockets and blood clots at the site of Vrana. Apamarg Kshara also possesses analgesic, antiinflammatory, hemostasis and antibacterial activity which offers symptomatic relief in Vrana.

Tikta \& Laghu Guna and Ushanya Virya of Haridra (Curcuma longa) provides Varnya, Sandhana, Vedanashamaka, Raktastambak, Raktashuddhikar and Lekhana properties. It enhances capillaries vasodilation when applied locally thus facilitates wound debridement (Shodhana) and wound healing (Ropana) process. It also possesses anti-inflammatory, analgesic and antibacterial activity.

\section{Conclusion}

- Ksharplota proved significantly effective in reducing Vrana Vedena, Vrana Daha, Vrana Kandu, Vrana Srava and Vrana Shotha.

- Ksharplota showed satisfactory outcome in healing of wound as compared to $\mathrm{H}_{2} \mathrm{O}_{2}+$ Eusol + Povidone application.

- The aim of the study was to assess the efficacy of Ksharaplota in the management of Dushta Vrana with the objective to give relief to patients of Dushta Vrana having sysmptoms like Vrana Vedena, Srava, Kandu, Daha Shotha. This objective was achieved both clinically and statistically.

- On the other hand Ksharaplota achieved statistically significant efficacy over modern Gold 
Anantkumar V Shekokar et.al., Study of the Efficacy of Ksharaplota in Dushtavrana w.S.r to Infected Wound

standard drugs used for dressing like $\mathrm{H}_{2} \mathrm{O}_{2}+$ Eusol

+ Povidone.

- Therefore it can be concluded that (Ksharaplota) is the effective with benefit of cost, free from hazard, easy procedure and tolerable to the patient

- Ksharaplota can be useful as first choice among Para surgical procedure in the management of Dushta Vrana (infected wound).

\section{References}

1. Sudarshana Shashtri \& Prof, Kaviratna Sharma, Original Text And Dalhana's Nibandhasangraha Commentary With Hindi Translation, R.A.V.Publication, New Delhi, 1'st Edition 2002 Sutrasthana, Adhyaya No.01, Sutra No.07, p-.05

2. Anantram Sharma, Sushruta Samhita Part-2, Choukhamba Surbharati Prakashan, Varanasi, 1st Edition, 2004, P-159.

3. Sudarshana Shashtri \& Prof, Kaviratna Sharma, Original Text And Dalhana's Nibandhasangraha Commentary With Hindi Translation, R.A.V.Publication, New Delhi, 1'st Edition 2002 Sutrasthana, Adhyaya No.22, Sutra No.07, p-.234.

4. Shekokar AV, Ukhalakar VP, Ayureveda Towards The Management Of Vrana W.S.R. To Approaches Of Shalayatantra : A Review, European Journal of Biomedical And Pharmaceutical Sciences, 2020;7(3);418-420.

5. Das S; A concise text book of surgery ; S. Das publication, Calcutta, $4^{\text {th }}$ edition, 1999, pg.no. 2.

6. Love And Bailey; Textbook Of Surgery; Edward Arnold (Publishers) Ltd; 16 ${ }^{\text {th }}$ Edition; New York; 2000; P- 34.

7. Love And Bailey; Textbook Of Surgery; Edward Arnold (Publishers) Ltd; 16 $6^{\text {th }}$ Edition; New York; 2000; P- 35.

8. Anantram Sharma, Sushruta Samhita Part-1, Chokhamba Surbharati Prakashan, Varanasi, 1st Edition, 2004, P-62, 69.

9. Sudarshana Shashtri \& Prof, Kaviratna Sharma, Original Text And Dalhana's Nibandhasangraha Commentary With Hindi Translation, R.A.V.Publication, New Delhi, 1'st Edition 2002, Pg.192.

10. Sudarshana Shashtri \& Prof, Kaviratna Sharma, Original Text And Dalhana's Nibandhasangraha Commentary With Hindi Translation, R.A.V. Publication, New Delhi, 1'st Edition 2002 Pg.130.s.

11. Shekokar AV, Borkar KM, Innovation Of Ksharaplota (Medicated Gauze) In the Management of Vrana (Ulcer), International Journal of Ayurveda and Pharama Research, 2013;1(2);10-16.

12. Sharma KR \& Kulwant Singh, Kshar-Sutra Therapy In Fistula In Ano And OtherAno-Rectal Disorders, Rastriya Ayurved Vidyapith Publication, New Delhi, 1st Edition, 1994, P-43.

13. Bhavprakash Nighantu, shri Bhramhashankar shastri, vidyotini hindi vyakhya, poorvardha, Page no.306, 415, 114, , Chaukhamba Sanskrit samsthan, Varanasi, $1^{\text {st }}$ edition 1984.

14. Shekokar AV, Borkar KM, Role of Ksharaplota In Lacerated Infected Wound - A Case Study, , International Journal of Ayurvedic Medicine, 2014;5(1);120-124.

15. Sudarshana Shashtri \& Prof, Kaviratna Sharma, Original Text And Dalhana's Nibandhasangraha Commentary With Hindi Translation, R.A.V.Publication, New Delhi, 1'st Edition 2002 Sutrasthana, Adhyaya No.11, Sutra No.05, pg-.102

16. Shekokar AV, Ukhalakar VP, Management of Infected Wound With Ksharaplota Dressing - A Case Study, Ayushdhara, 2018;5(2);1629-1634.

17. Shekokar AV, Ukhalakar VP, Critical Review on Ksharaplota and its Therapeutics Aspects, Journal of Drug Delivery and Therapeutics.2019;9(1s);510-515.

18. Sharma KR \& Kulwant Singh, Kshar-Sutra Therapy In Fistula In Ano And OtherAno-Rectal Disorders, Rastriya Ayurved Vidyapith Publication, New Delhi, $1^{\text {st }}$ Edition, 1994, P-53.

19. Sharma KR \& Kulwant Singh, Kshar-Sutra Therapy In Fistula In Ano And OtherAno-Rectal Disorders, Rastriya Ayurved Vidyapith Publication, New Delhi, $1^{\text {st }}$ Edition, 1994, P-51.

20. Pande DN, Anushastra Karma Parasurgical Therapy at a Glance, Chaukhambha Visvabharati Publication, Varanasi, 1st edition, 2009, pg.49.

21. Sharma KR \& Kulwant Singh, Kshar-Sutra Therapy In Fistula In Ano And OtherAno-Rectal Disorders, Rastriya Ayurved Vidyapith Publication, New Delhi, $1^{\text {st }}$ Edition, 1994, P-54.

22. Shekokar AV, Ukhalakar VP, Formulation and Physico-Chemical Characterization of Ksharaplota Dressing for Vrana Chikitsa, International Journal of Ayurveda and Pharama Research, 2018;6(7);44-50.

23. Shekokar AV, Ukhalakar VP, Expository aspect for action of Ksharaplota, Journal of Drug Delivery and Therapeutics.2018;8(6-s);420-423. 